Discussion The mortality rate among this population of patients with tuberculosis far exceeds that in the UK. Sadly a lake of resources, HIV coinfection and high rate of drug resistance $(10 \%$ $\mathrm{MDR}$ rate) conspire to make $\mathrm{TB}$ a challenging disease to treat in this area of South Africa.

\section{Respiratory critical care P66 INTER-OBSERVER RELIABILITY OF ULTRASOUND TO MEASURE RECTUS FEMORIS CROSS-SECTIONAL AREA IN CRITICALLY ILL PATIENTS}

doi:10.1136/thoraxjnl-2011-201054c.66

\begin{abstract}
1,2B Connolly, ${ }^{1,2,3} \mathrm{Z}$ Puthucheary, ${ }^{3} \mathrm{H}$ Montgomery, ${ }^{1} \mathrm{~J}$ Moxham, ${ }^{1,2} \mathrm{~N}$ Hart. ${ }^{1}$ Department of Asthma, Allergy \& Respiratory Science, Division of Asthma, Allergy and Lung Biology, King's College London, London, UK; 'uy's \& St Thomas' NHS Foundation Trust and King's College London, National Institute of Health Research Comprehensive Biomedical Research Centre, London, UK; ${ }^{3}$ Institute of Health and Human Performance, University College London, London, UK
\end{abstract}

Introduction Ultrasound is a relatively simple, non-invasive, nonirradiating effort-independent tool to measure quadriceps rectus femoris cross-sectional area $\left(\mathrm{RF}_{\mathrm{CSA}}\right)$ in critically ill patients. We investigated the inter-observer reliability of the technique to validate its clinical utility in this group of patients.

Methods Critically ill patients either in, or within $48 \mathrm{~h}$ discharge from, the Intensive Care Unit (ICU) underwent measurement of $\mathrm{RF}_{\mathrm{CSA}}$ using real-time B-mode ultrasonography using an $8 \mathrm{MHz}$ $5.6 \mathrm{~cm}$ linear transducer (PLM805, Toshiba Medical Systems Ltd, Crawley, UK) at a distance three-fifths from the anterior superior iliac spine to the superior patellar border. Where complete visualisation of $\mathrm{RF}_{\mathrm{CSA}}$ was not possible at this point, a more distal point of $2 / 3$ of this distance was used. Ultrasound measurements were performed in turn by two critical care clinicians trained in ultrasound in a random order. The average of three consecutive measurements within $10 \%$ was taken as $\mathrm{RF}_{\mathrm{CSA}}$ for each patient. Both clinicians were blinded to the results of the other.

Results 24 patients had $\mathrm{RF}_{\mathrm{CSA}}$ measurements performed using ultrasound (M:F 14:10; mean age 55.3 \pm 20.1 years). Inter-observer reliability was assessed by considering the level of agreement between $\mathrm{RF}_{\mathrm{CSA}}$ measurements for each patient between the two clinicians using intra-class correlation coefficients (ICC) adopting a two-way, random effects model for absolute agreement. An ICC of 0.99 (95\% CI 0.97 to 0.99) was observed. Abstract P66 Figure 1 shows $\mathrm{RF}_{\mathrm{CSA}}$ images from both clinicians for one patient.
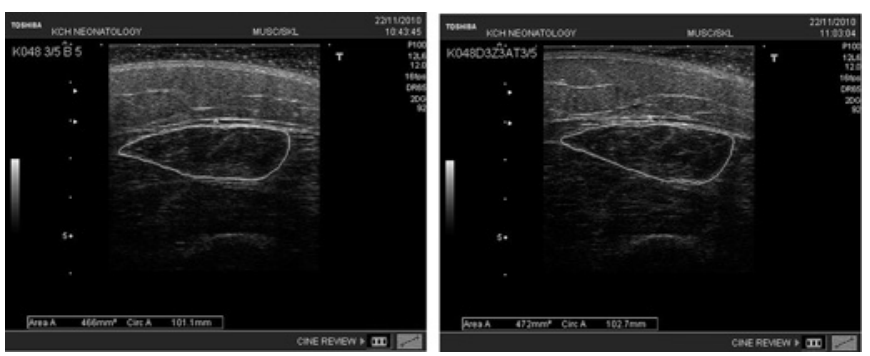

Abstract P66 Figure 1 Ultrasound images from each clinician for one patients; $\mathrm{RF}_{\mathrm{CSA}}$ outlined in blue.

Conclusion These data demonstrate high levels of inter-observer reliability between two trained critical care clinicians using ultrasound as a measurement technique for $\mathrm{RF}_{\mathrm{CSA}}$ in critically ill patients. $\mathrm{RF}_{\mathrm{CSA}}$ can be used as a novel, reproducible technique to track the trajectory of muscle loss in critically ill patients. P67 CLINICAL PREDICTIVE VALUE OF THE MEDICAL RESEARCH
COUNCIL SUMSCORE IN CRITICALLY ILL PATIENTS

doi:10.1136/thoraxjnl-2011-201054c.67

${ }^{1,2} \mathrm{~B}$ Connolly, ${ }^{3} \mathrm{~A}$ Curtis, ${ }^{3} \mathrm{G}$ Jones, ${ }^{1} \mathrm{P}$ Murphy, ${ }^{1} \mathrm{~J}$ Moxham, ${ }^{1,2} \mathrm{~N}$ Hart. ${ }^{1}$ Department of Asthma, Allergy \& Respiratory Science, Division of Asthma, Allergy and Lung Biology, King's College London, London, UK; ' $G u y$ 's \& St Thomas' NHS Foundation Trust and King's College London, National Institute of Health Research Comprehensive Biomedical Research Centre, London, UK; ${ }^{3}$ Physiotherapy Department, St. Thomas' Hospital, Guy's \& St. Thomas' NHS Foundation Trust, London, UK

Introduction Manual muscle testing, in the form of the Medical Research Council sumscore (MRC-SS) is a widely accepted clinical tool for diagnosing intensive care unit-acquired weakness (ICUAW). Although MRC-SS is a simple bedside test, the nature of the test is volitional limiting its ability to distinguish poor motivation and impaired cognition from actual loss of muscle function. The clinical predictive value of the MRC-SS therefore needs to be assessed.

Method Unselected adult ICU patients (=18 years) ventilated for $=48 \mathrm{~h}$ were eligible. The conscious level of the patients was determined using the Richmond Agitation Sedation Scale; a score -1 to +1 was indicative of awakening. Testing comprised of a two-stage process. Stage 1: Patients at awakening were required to follow 4 simple, one-stage commands. Stage 2: If all 4 one-stage commands were successfully completed, MRC-SS testing was performed by a specialist ICU rehabilitation clinician. ICU-AW was defined as MRC-SS $<48$. ICU and hospital mortality and length of stay (LOS) were recorded in all the patients.

Results 94 sequential awakening patients were recruited; $68.1 \%$ males $(n=64)$, with a mean age for the whole cohort of $64.5 \pm 15.3$ years. 29 patients were unable to successfully complete the 4 one-stage commands as a result of cognitive impairment. 65 patients completed the MRC-SS of whom $73.9 \%$ demonstrated ICU-AW at awakening. Results are shown in Abstract P67 table 1.

Abstract P67 Table 1 Summary of test characteristics of MRC-SS

\begin{tabular}{|c|c|c|c|c|c|c|c|c|}
\hline \multirow{2}{*}{$\begin{array}{l}\text { Test } \\
\text { characteristic }\end{array}$} & \multicolumn{2}{|c|}{ ICU mortality } & \multicolumn{2}{|c|}{$\begin{array}{l}\text { ICU LOS } \\
\text { ( } \leq 14 ;>14 \text { days })\end{array}$} & \multicolumn{2}{|c|}{$\begin{array}{l}\text { Hospital } \\
\text { mortality }\end{array}$} & \multicolumn{2}{|c|}{$\begin{array}{l}\text { Hospital LOS } \\
(\leq 28 ;>28 \text { days })\end{array}$} \\
\hline & UTC & ICU-AW & UTC & ICU-AW & UTC & ICU-AW & UTC & ICU-AW \\
\hline Sensitivity & 0.69 & 0.88 & 0.22 & 0.93 & 0.56 & 0.81 & 0.17 & 0.84 \\
\hline Specificity & 0.84 & 0.28 & 0.64 & 0.41 & 0.84 & 0.29 & 0.56 & 0.41 \\
\hline PPV & 0.62 & 0.15 & 0.28 & 0.54 & 0.69 & 0.27 & 0.28 & 0.67 \\
\hline NPV & 0.88 & 0.94 & 0.57 & 0.88 & 0.75 & 0.82 & 0.42 & 0.65 \\
\hline
\end{tabular}

UTC $(n=29)$. MRC-SS $<48(n=48)$. MRC-SS $\geq 48(n=17)$.

ICU, Intensive Care Unit; ICU-AW, intensive care unit-acquired weakness; LOS, length of stay; NPV, negative predictive value; PPV, positive predictive value; UTC, unable to complete 4 one-stage commands.

Conclusion Almost a third of critically ill patients, from a sequential cohort, were unable to complete 4 one-stage commands and thus could not perform the MRC-SS. Although inability to successfully complete the one-stage commands conferred limited predictive value, those patients that could perform this task were more likely to survive ICU. Similarly an MRC-SS $<48$ at awakening, presumed indicative of ICU-AW, conferred limited predictive value. However an MRC-SS $=48$ predicted ICU and hospital survival as well as an ICU LOS $<2$ weeks. These data highlight the limitations of volitional tests in critically ill patients. It clearly challenges the current view that ICU-AW, as measured by volitional tests, is a predictor of poor outcome. These data confirm that preserved peripheral strength predicts a good outcome. 